\title{
Study on Combination Model of Wind Power Generation Prediction
}

\author{
LIU Guan-qi, HU Ting, SHAO Long \\ North China Electrical Power University, Baoding, China, 071000 \\ jane_ms@126.com
}

\begin{abstract}
With the installed wind capacity increasing rapidly, the security, stability and economic operation of the power grid have been influenced because of the randomness and volatility of the wind power. Wind power prediction is an effective approach for the above problems. This article is about the theory of combination forecast and establishes two combination forecast models by combining RBF network power prediction model, time series model and support vector machine (SVM) model. Finally, through comparative analysis of the results, combination model can get better prediction accuracy, and better meets the actual needs.
\end{abstract}

Keywords- wind power prediction; neural work; time series; support vector machine; combined forecasting

\section{INTRODUCTION}

With the rapid increase of installed capacity of wind generation and the construction of large scale wind farms, the power grid of every country pay more attention to wind power [1]. The short-term prediction of wind speed and wind power generation for wind integration and grid dispatching plays a vital role. Currently, mathematical models of wind prediction can be classified into: time series model, neural network model and SVM (support vector machine) model. Single prediction models have their own advantages and disadvantages and can affect the prediction result differently because of their different raw data, so that it is not a mutually exclusive relationship but a compensate one among the single models. Therefore, the combined forecasting method in wind farms has taken more and more attention.

\section{RBF NEURAL NETWORK MODEL BASED ON PHASE SPACE RECONSTRUCTION [2]}

RBF (Radial Basis Function) is a kind of feed forward neural network with single hidden layer, it has been verified that RBF neural network can approximate arbitrary continuous function with arbitrary precision.

Affected by wind speed, wind direction and other weather element, the power output of wind farms presents a sort of chaos. The first step to analyze chaotic dynamic system is to reconstruct phase space. According to the embedding theorem presented by Takens, evolution of any component is determined by components interacting with it, so that phase space reconstruction takes only one component into consideration. Since we can find a m-dimensional vector through some fixed point data, it can reconstructed a new phase space that is equivalent to the original one. As for chaotic time series $x 1, x 2, \cdots, x n$, it's important to chose proper embed dimension and time delay. Set the two parameters $m$ and $\tau$ respectively, the reconstructed phase space can be described as formula (1).

$$
\begin{aligned}
& Y m(i)=[x(i), x(i+\tau), x(i+2 \tau), \cdots, x(i+(m-1) \tau)] \\
& (i=1,2, \cdots, N ; N=n-(m-1) \tau)
\end{aligned}
$$

\section{TIME SERIES MODEL [3]}

As for time series sequence $\{x i\}$, three models can be used to describe it: autoregressive (AR) model, moving average (MA) model and autoregressive moving average (ARMA) model. The third model has characteristics of both AR(p) model and MA(q) model, and $\{x i\}$ can be described as the following formula $(2)$.

$$
x i=\phi 1 x i-1+\cdots+\phi p x i-p+\varepsilon i-\theta 1 \varepsilon-1-\cdots-\theta q \varepsilon i-q
$$

Where $\{\varepsilon\}$ presents noise series.

Since $\operatorname{ARMA}(\mathrm{p}, \mathrm{q})$ model can only describe stationary time series, most of the time the raw time series need $d$ order difference disposal. Therefore, with the operator, the mixed model ARIMA(p,d,q) is formed and it embody obvious advantages at prediction issues.

\section{SVM(SUPPort Vector MACHINE) MOdEL [4]}

SVM's core is that it uses nonlinear transformation defined by inner product function to transform input space to higher space, and generalized optimal separate hyperplane can be work out. At present the theory is still in the development, in the article least squares support vector machine (LS-SVM) is adopted.

For sample space $(x 1, y 1),(x 2, y 2), \cdots,(x l, y l)$, firstly a nonlinear mapping $\varphi(\cdot)$ is needed to be constructed between sample space and characteristic space $\varphi\left(x_{i}\right)$, and then optimal decision function will be established in the high dimensional space as follow:

$$
y(x)=\omega \varphi(x)+b
$$

So nonlinear estimation function could be converted to linear estimation function which belongs to high dimensional space, and then it calculates the minimum of $\omega 、 b$ according to Structural Risk Minimization so that:

$$
\left[\begin{array}{cc}
0 & \mathbf{I}^{\mathrm{T}} \\
1 & \mathbf{Z Z}^{\mathrm{T}}+\gamma^{-1} \mathbf{I}
\end{array}\right]\left[\begin{array}{l}
b \\
\boldsymbol{a}
\end{array}\right]=\left[\begin{array}{l}
0 \\
\mathbf{y}
\end{array}\right]
$$

Where $\mathbf{y}=[y 1, y 2, \cdots, y l]^{\mathrm{T}} ; \quad \boldsymbol{\alpha}=\left[\alpha_{1}, \alpha 2, \cdots, \alpha l\right]^{\mathrm{T}} ; \quad \mathbf{z Z}^{\mathrm{T}}$ is a square matrix of $l \times l ; \mathbf{Z}=[\varphi(x 1), \varphi(x 1), \cdots, \varphi(x 1)]^{\mathrm{T}} ; \quad$ The element of $\mathrm{m}$ line and n column is $\varphi(x m) \varphi\left(x_{n}\right)$ 。

It adopts kernel function $K\left(x, x_{i}\right)$ to take the place of $\varphi(x m) \varphi(x n)$ and transform dot product in high dimensional characteristic space to kernel function to make calculation. 
$a$ and $b$ can be figured by formula(3), regression analysis consequence of nonlinear function could be described as:

$$
f(x)=\sum_{i=1}^{l} \alpha_{i} K(x, x i)+b
$$

LS-SVM improves the training speed of traditional SVM observably; especially it is more efficient to solve the problem of large-scale data calculation

\section{LINEAR COMBINED FORECASTING MODEL}

In 1969, Bates and Granger first proposed the concept of combined forecasting mode described as "Synthesizing the characteristics of each single prediction method and the information provided by each method, combined forecasting model is to combine different single prediction method together with appropriate weight". Combined forecasting method for wind power prediction is at the very beginning and is the future research direction.

As for linear combined forecasting model, supposing $\mathrm{m}$ is the number of single prediction models and $f_{i}$ is the predicted value of the ith model, the linear combined forecasting model is described as $f=l_{1} f_{1}+l_{2} f_{2}+\cdots+l_{m} f_{m}$, where $l 1, l 2, \cdots l m$ present the weight of each single prediction model, respectively. The key problem of establishing combined forecasting model is to determine weight of each single model. The common approaches to get weighting coefficients are as follows.

\section{A. least-squared-error criterion [5]}

Suppose $e_{i}$ and $\operatorname{Var}\left(e_{i}\right)$ are respectively absolute error and variance of the ith single prediction method, while $e$ and $\operatorname{Var}(e)$ are respectively absolute error and variance of the combined forecasting model, so that they can be described as formula(5)and formula(6)

$$
\begin{gathered}
e=\sum_{i=1}^{m} \text { liei } \\
\operatorname{Var}(e)=\operatorname{Var}\left(\sum_{i=1}^{m} l i e i\right)=\sum_{i=1}^{m} l_{i}^{2} \operatorname{Var}(e i)+2 \sum_{i=1}^{m} \sum_{j=1}^{m} l i l j \operatorname{Cov}\left(e \mathrm{e}, \mathrm{e}_{\mathrm{j}}\right)
\end{gathered}
$$

Take minimum variance as optimum target, the weight coefficients can be obtained by the following formula.

$$
l_{i=\frac{\sum_{k=1, k \neq i}^{m}}{} \sum_{k=1, j \neq i, k}^{m} \operatorname{Var}(e k) \operatorname{Var}\left(e_{j}\right)}^{\sum_{j=1, j \neq k}^{m} \sum_{i=1,2, \cdots, m}^{m} \operatorname{Var}(e k) \operatorname{Var}\left(e_{j}\right)} \quad i=
$$

B. Optimal non-negative variable weighting coefficient method [6]

This is a variable-weight combined method. Forecast object $\{x t, t=1,2, \cdots, N\}$ is described as $\hat{x} t=\sum_{t=1}^{m}$ litxit .

Let $e t$ and $e i t=x t-x i$ be respectively the absolute error of the combined model and the ith single prediction model at time $t$, the combined forecasting model of non-negative variable weighting coefficient can be described as an optimization problem shown in the following formula $(8)$.

$$
\begin{aligned}
& \min J=\sum_{t=1}^{N} \sum_{i=1}^{m} \sum_{j=1}^{m} \text { litljteitejt } \\
& \text { s.t. }\left\{\begin{array}{l}
\sum_{t=1}^{m} \text { lit }=1, \quad t=1,2, \cdots, N, \\
l i t \geq 0, \quad i=1,2, \cdots, m, t=1,2, \cdots, N
\end{array}\right.
\end{aligned}
$$

Let $\mathbf{L} t=(l 1 t, l 2 t, \cdots, l m t)^{\mathrm{T}}, \mathbf{E} t=(e 1 t, e 2 t, \cdots, e m t)^{\mathrm{T}}$ so $e t=\mathbf{L} t^{\mathrm{T}} \mathbf{E} t$ and $e t^{2}=\mathbf{L} t^{\mathrm{T}} \mathbf{E} t \mathbf{E} t^{\mathrm{T}} \mathbf{L} t$ where $\mathbf{E} t \mathbf{E} t^{\mathrm{T}}$ presents the covariance matrix of the prediction error at time t. Set non-negative variable weighting coefficient vector $\mathbf{L}=\left(\mathbf{L}_{1}{ }^{\mathrm{T}}, \mathbf{L} 2^{\mathrm{T}}, \cdots, \mathbf{L} m^{\mathrm{T}}\right)^{\mathrm{T}}$, so that the prediction error's covariance matrix of the $\mathrm{m}$ single prediction models can be described as formula (9).

$$
\mathbf{E}=\left[\begin{array}{llll}
\mathbf{E} 1 \mathbf{E} 1^{\mathrm{T}} & & & \\
& \mathbf{E} 2 \mathbf{E} 2^{\mathrm{T}} & & \\
& & \ddots & \\
& & & \mathbf{E} N \mathbf{E} N^{\mathrm{T}}
\end{array}\right]
$$

So that, the sum square error can be expressed as $J=\mathbf{L}^{\mathrm{T}} \mathbf{E} \mathbf{L}$. Let $\mathbf{R}=(\mathbf{0}, \mathbf{0}, \cdots, \mathbf{R} t, \cdots, \mathbf{0})_{1 \times m N}$ and the formula (5-11) is further expressed as formula(10)

$$
\begin{array}{ll}
\min & J=\mathbf{L}^{\mathrm{T}} \mathbf{E} \mathbf{L} \\
\text { s.t. } & \left\{\begin{array}{l}
\mathbf{R} t^{\mathrm{T}} \mathbf{L}=1, \quad t=1,2, \cdots, N, \\
\mathbf{L} \geq \mathbf{0} .
\end{array}\right.
\end{array}
$$

The weight coefficient $\mathrm{L}$ of the linear programming problem can be worked out by the formula (10)

\section{APPLICATION EXAMPLE}

\section{A. Application of the three signal prediction models}

In a wind farm in Inner Mongolia of China, chose the generated power sequence from May 29th, 2011 to June $27 \mathrm{th}, 2011$ and its sample rate is 10 minute, so the time series include 4320 points which record temporal power data. Similarly, construct time series of wind speed data. Then utilize the data of previous 29 days to forecast the data of the 30 th day with programming function of MATLAB.

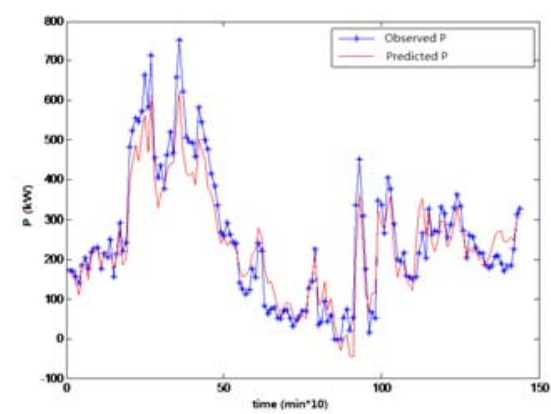

Figure 1. Prediction result by RBF model 


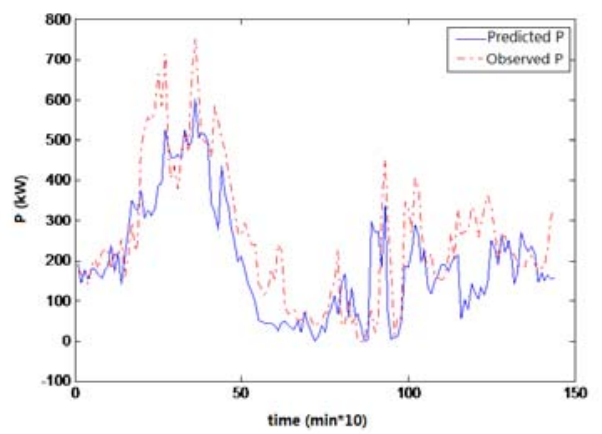

Figure 2. Prediction result by time series model

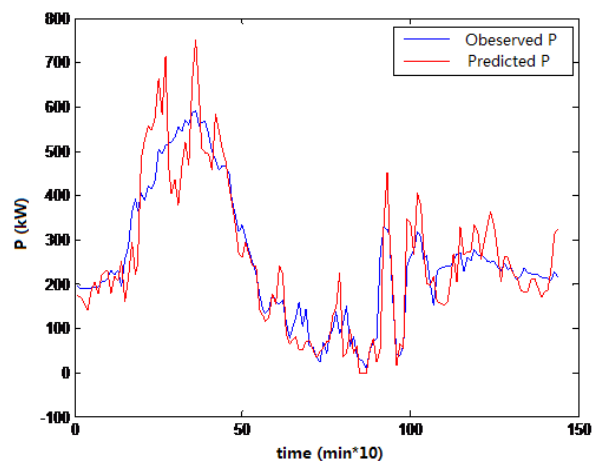

Figure 3. Prediction result by LS-SVM model

Fig. 1, Fig. 2 and Fig. 3 are respectively the results of prediction by RBF neural network model, time series model and LS-SVM model.

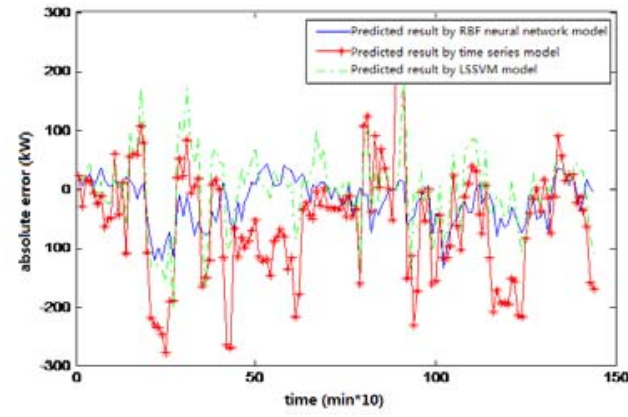

Figure 4. Error comparation of the three single models

As shown in Fig.4, prediction accuracy of neural network model based on chaos theory is more accurate; Time series model firstly make wind speed forecast by original wind speed data, and then short-term power forecast is obtained by wind power generation output model, so its forecast error is biggish; Forecast consequence of LS-SVM is more accurate than time series model. The first two methods are more adaptive for complex short-term power forecast in wind farm.

\section{B. Application of two combined forecasting models}

According to the results of the three single prediction models, suppose eRBF, eARIMA and eLSSVM are respectively the errors of the single models; IRBF , LARIMA and ILSSVM are the weight coefficients. This paper uses least-squared-error criterion and optimal non-negative variable weighting coefficient method to compute the coefficients so that combined forecasting model I and combined forecasting model II are formed, respectively.

Combined forecasting model I : The coefficients values are calculated as IRBF $=0.6830, \quad$ IARIMA $=0.1021$ and $L_{L S S V M}=0.2149$ respectively. RMS error and percentage error of this model are respectively 46.6045 and 0.0269 . Fig. 5 is the result of combined prediction model I .

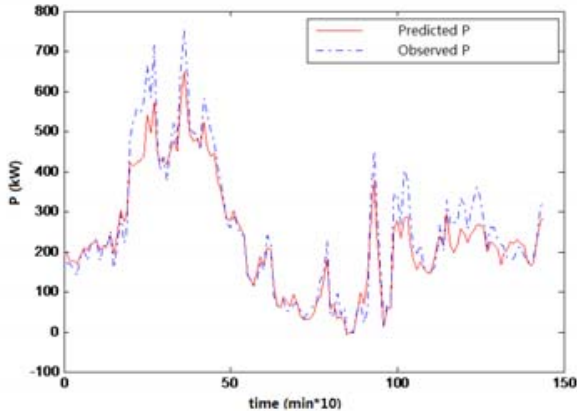

Figure 5. Prediction result by combined model I

Combined prediction model II: The coefficients are obtained by formula(12) and the prediction results can be obtained by formula(9). Fig.6 shows the prediction result; RMS error and percentage error are 23.7537 and 0.0144 .

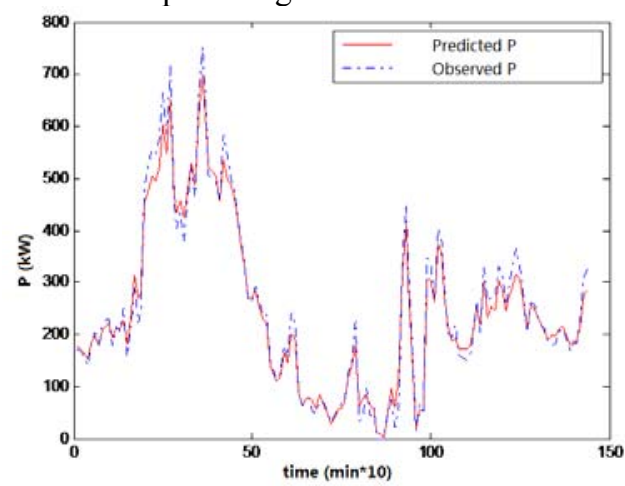

Figure 6. Prediction result by combined model II

\begin{tabular}{|c|c|c|c|c|c|}
\multicolumn{2}{c}{ TABLE I } & \multicolumn{3}{c|}{ ERROR COMPARATION OF THE MODELS } \\
\hline Enor type & $\begin{array}{c}\text { RBF } \\
\text { model }\end{array}$ & $\begin{array}{c}\text { ARIMA } \\
\text { model }\end{array}$ & $\begin{array}{c}\text { LSSVM } \\
\text { model }\end{array}$ & $\begin{array}{c}\text { combined } \\
\text { model I }\end{array}$ & $\begin{array}{c}\text { combined } \\
\text { model II }\end{array}$ \\
\hline $\boldsymbol{e}_{R M S E, P}$ & 53.846 & 112.498 & 67.282 & 46.6045 & 23.7537 \\
\hline $\boldsymbol{e}_{R M E, P}$ & 0.0348 & 0.0687 & 0.0404 & 0.0269 & 0.0144 \\
\hline
\end{tabular}

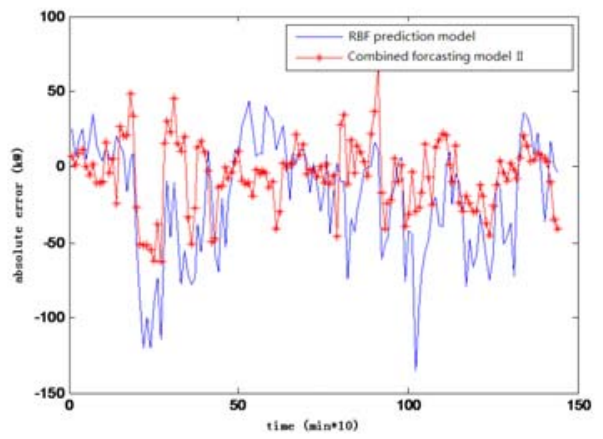

Figure 7.Errorcomparation of combinedmodel II andRBF model 
Table 1 compares the root mean square error and percentage error of each prediction model. Fig. 7 shows the comparison of combined forecasting model II and RBF neural network model which is the most accurate among single models. From Fig. 7 and Table 1, it can be seen that combining three single prediction models together combined prediction model 1 and combined prediction model 2 both obtain more accurate prediction results. Furthermore, combined model 2 is more accurate and practical than combined model 1 .

\section{SUMMARY}

By analyzing prediction results of different models, it can be seen that each single prediction method has its own advantages and disadvantages; they share a relationship that is not mutually exclusive but complementary with each other. If only one method is used for prediction, the prediction result can only reflect some of the elements that affect the predicted results, so that information of other elements else is lost and it will be quite important and not easy to chose a appropriate prediction single model. In the practical prediction work, combining some single prediction models together to form a combined forecasting model will increase the accuracy and reliability of the prediction. As mentioned above, combined forecasting method will arouse more and more interest and have a wide prospect for research and application.

\section{REFERENCES}

[1] LEI Yazhou. Studies on wind farm integration into power system. Automation of Electric Power Systems, 2003, 27(8):84-88.

[2] PINSON P, KARINIOTAKIS G N. Wind power forecasting using fuzzy neural networks enhanced with on-line prediction risk assessment// IEEE Power Tech Conference Proceedings:Vol 2, June 23-26, 2003, Bologna, Italy: 8p..

[3] DING Ming, ZHANG Lijun, WU Yichun. Wind speed forecast model for wind farms based on time series analysis. Electric Power Automation Equipment, 2005, 25(8): 32-34.

[4] M.A. Mohanders, T.O. Halawani, S.Rehman, Ahmed A. Hussain. Support vector machines for wind speed prediction [J]. Renewable Energy, 2004, 29(16): 939-947.

[5] ZHANG Guoqiang, ZHANG Boming. Wind speed and wind turbine output forecast based on combination method [J]. Automation of Electric Power System, 2009, 33(18):93-95.

[6] Ma Yongkai, Tang Xiaowo, Yangguiyuan. A study on basic theory of the optimal combinated prediction method of non-negative weights $[\mathrm{J}]$. Operations Research And Management Science, 1997,6(2):1-8. 